\title{
Formación inicial docente en igualdad de género, evidencias desde los programas de estudio
}

\author{
Initial teacher formation in gender equality, evidence derived \\ from the study programs
}

ISSN 2071-8748

E-ISSN 2218-3345

(c) (1) (2)

BY NC SA

URI: http://hdl.handle.net/11298/875

DOI: http://dx.doi.org/10.5377/entorno.v0i66.6725
Lorenzo Aravena-Ramírez daniel.aravena1881@gmail.com Universidad Católica del Maule, Chile ORCID: 0000-0003-0964-0437

Marta Belmar-Mellado mbelmar@ucm.cl Universidad Católica del Maule, Chile ORCID: 0000-0002-1326-078X

Esta investigación fue financiada por el Programa Formación de Capital Humano Avanzado de la Comisión Nacional de Investigación Científica y Tecnológica (Conicyt/21170343).

Recibido: 30/07/18

Aprobado: 12/12/18

\section{Resumen}

La incorporación de la igualdad de género en los planes de estudio de la formación de los futuros profesores es una estrategia que buscar desarrollar en ellos una visión positiva hacia este tema, para que así al momento de egresar y desarrollar sus clases, lo expresen frente a sus estudiantes $y$ al seleccionar sus actividades de aprendizaje. Por eso se ha querido indagar en los programas de formación de la Carrera de Pedagogía en Educación General Básica con mención, impartida por la Universidad Católica del Maule, en Chile. Esta institución plantea que forma a sus profesionales bajo un modelo basado en tres tipos de competencias: Generales, Profesionales y Disciplinares, por lo tanto, el itinerario formativo de esta carrera está enfocado a desarrollar las competencias, a través de sus 56 programas de estudio. La metodología utilizaba se sustenta en un paradigma interpretativo, con enfoque epistemológico cualitativo y, la técnica de recolección de la

\section{Abstract}

The incorporation of gender equality into the study plans and into the formation of future teachers is a strategy that seeks to develop in them a positive vision towards this issue, so after graduating and when teaching and choosing different learning activities it can be perceived by their students. That is why, the study programs of the Elementary Education Pedagogy career of the Universidad Católica del Maule (Catholic University of the Maule), Chile have been a subject of study. This institution sets out that professionals are formed under a model based on three types of competencies: general, professional and disciplinary, therefore, the formative itinerary of this bachelor focuses on developing competencies through its 56 study programs. The methodology used was based on an interpretative paradigm, with a qualitative epistemological approach, and the technique of gathering information was through documentary based analysis. 
información fue a través del análisis documental. Se realizó la búsqueda que 21 conceptos relacionados con la igualdad de género, utilizando para ello el software NVivo v. 12, en cada uno de los programas que tributan a cada una de las competencias de formación declaradas. Los resultados muestran una baja presencia de los conceptos relacionados con igualdad de género y a su vez evidencian una diferencia entre la presencia del concepto de mujer y hombre, ya que este último predomina más veces que el concepto de mujer.

\section{Palabras clave}

Derecho a la educación; Igualdad en la educación; Formación profesional; Efectividad del docente.
The search of 21 concepts related to gender equality was carried out using NVivo 12 software in each of the formative competencies stated. The results show a low presence of concepts related to gender equality, which in turn evinces a difference between the concepts woman and man since the latter predominates more times than that of woman.

\section{Keywords}

Right to education, Equality in education, Professional formation, Teacher effectiveness.

\section{Introducción}

La investigación desarrollada, ha tenido por objetivo abordar el tema de la inclusión del tema de igualdad de género en los programas de estudio de la carrera de Pedagogía en Educación General Básica con mención (PEGB), impartida por la Universidad Católica del Maule (UCM), de Chile, y para lograrlo primeramente se indagó en el modelo formativo, donde se describe que esta institución presenta una formación basada en tres tipos de competencias: generales, profesionales y disciplinares.

En los últimos 10 años, el ingreso de estudiantes a esta carrera ha sido mayoritariamente femenino, como se puede observar la figura 1. Por lo tanto, la temática de igualdad de género resulta relevante de ser investigada, ya que estos profesionales serán los que eduquen a niños y niñas de entre 6 y 14 años, que serán parte de la sociedad, pudiendo ser agentes de cambio.

Figura 1. Ingreso de estudiantes a la carrera de Pedagogía en Educación General Básica, por año de ingreso y género

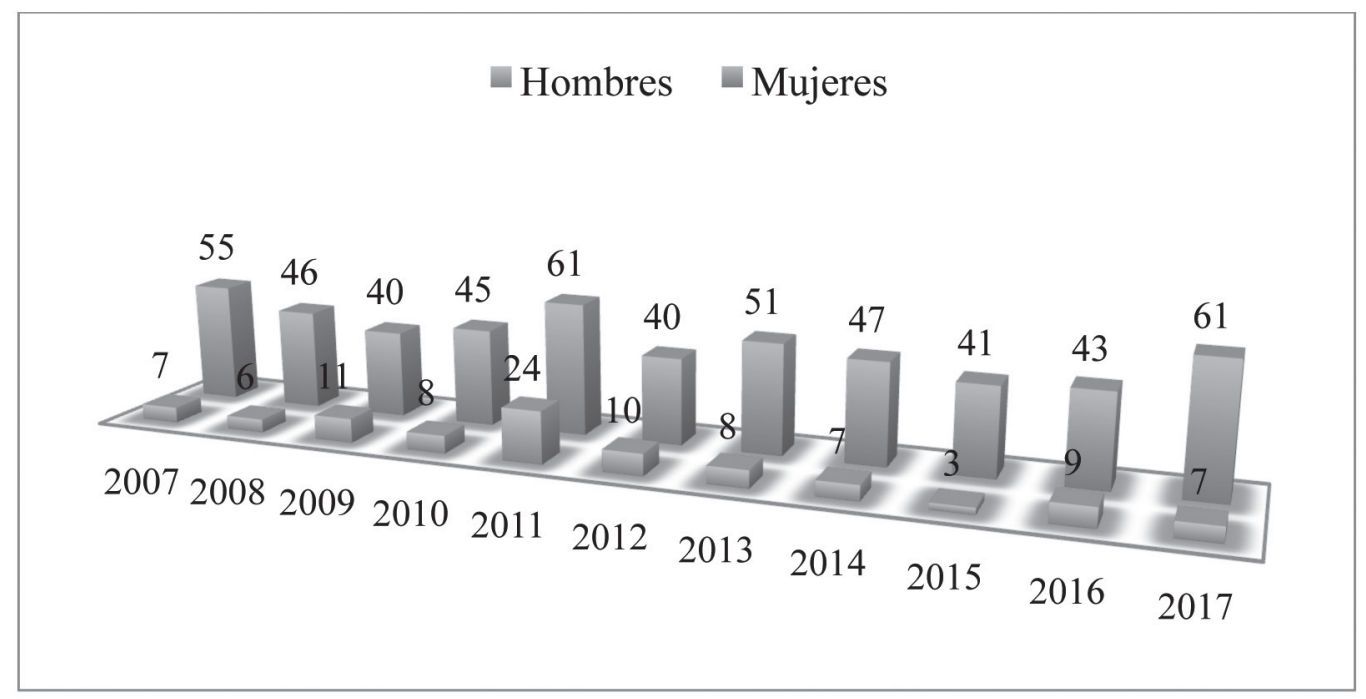


La investigación se encuentra organizada en tres apartados: 1) referente a la metodología que se utilizó y la forma en que fue realizada la recolección de datos, que en este caso fue mediante análisis documental para ver la inclusión de conceptos de igualdad de género dentro de los 56 programas de estudio, de la carrera de PEGB, 2) presentación de los resultados, que permite tener una visión de cuál es la presencia que tienen los conceptos relacionados con la igualdad de género dentro de los programas de estudio relacionados a los tres tipos de competencias que plantea esta universidad como base para la formación de sus profesionales. En este sentido, la investigación realizada es importante porque aporta resultados acerca de la presencia de dichos conceptos dentro de los programas de estudio de una carrera que forma a los profesores, quienes serán parte del nivel educacional más extenso de Chile; y 3) presentación de más conclusiones a las que permiten llegar los resultados de esta investigación, y además, finalmente, se realizan algunas sugerencias de futuras líneas de investigación sobre el tema de la igualdad de género y la formación inicial docente. Estas buscan resaltar la importancia que tiene la educación en el desarrollo de la igualdad de género, ya que así este tema se hace una más visible con el objetivo de generar condiciones de igualdad para hombres y mujeres en la sociedad,

\section{Metodología}

Esta investigación se inscribe bajo un paradigma interpretativo (Serrano, 2016), puesto que no busca explicaciones casuales de la vida social y humana, sino profundizar el conocimiento y comprensión del porqué de una realidad (Torres, 2016). También es descriptivo porque busca exponer cómo se desarrolla el tema de la igualdad de género dentro de los programas de estudio de la carrera de PEGB de la universidad. El enfoque epistemológico, como señala Bisquerra (2016), será cualitativo, ya que no busca la generalización, sino que es ideográfica y se caracteriza por estudiar en profundidad una situación concreta. Se utilizó la técnica de análisis documental (Pinto, Gálvez, \& Dijk, 2018) para tener una visión más amplia y exhaustiva de los tópicos considerados en los programas de formación pedagógica de la carrera en cuestión.

El acceso al corpus documental se ha hecho mediante una muestra no probabilística, donde se plantea que la elección de los elementos no depende de la probabilidad, sino de causas relacionadas con las características de la investigación (Hernández, Fernández \& Baptista (2014). En este caso las características que establecen relación son las competencias bajo las cuales se forma a los profesores y profesoras de Educación General Básica con mención. La UCM desarrolla la formación de profesionales bajo tres tipos de competencias:

- Competencias generales

- Competencias disciplinares

- Competencias profesionales

Por lo tanto, el itinerario formativo de los estudiantes de PEGB con mención está distribuido de la siguiente forma:

Tabla 1. Descripción de la cantidad de programas relacionados con cada competencia de formación declarada por la universidad

\begin{tabular}{|l|c|}
\hline Competencias & $\begin{array}{c}\text { Número de } \\
\text { programas }\end{array}$ \\
\hline General & 7 \\
\hline Disciplinares & 21 \\
\hline Profesionales & 28 \\
\hline
\end{tabular}

El tipo de análisis utilizado fue por conglomerados (Casal, \& Mateu, 2003), ya que utiliza como unidades de análisis los grupos de elementos que presentan asignaciones y límites naturales. Para el caso de este estudio, son los programas de estudio impartidos según los tres tipos de competencias en los que se forman los profesionales en esta universidad (Krippendorff, 1990). Seguidamente, se realizó un análisis de los programas de estudio de la carrera de PEGB con mención, tomando en cuenta las competencias para identificar 21 conceptos relacionados con la igualdad de género dentro de los programas de estudio que son parte del itinerario formativo de esta carrera.

Los conceptos estudiados sobre igualdad de género surgen a partir de la búsqueda realizada por Rebollar (2013), para el desarrollo de su tesis doctoral, donde investigó sobre la temática en la formación de profesores. A partir de esto se elaboró una base de datos, con los programas, la cual fue introducida en el programa NVivo v,12 donde se realizó la búsqueda de la ocurrencia de los diferentes conceptos mencionados anteriormente (ver tabla 2). 
Tabla 2. Categorías de análisis con de conceptos relacionados con igualdad de género

\begin{tabular}{|c|c|c|c|c|c|}
\hline \multicolumn{2}{|c|}{ Competencias genéricas } & \multicolumn{2}{|c|}{ Competencias profesionales } & \multicolumn{2}{|c|}{ Competencias disciplinares } \\
\hline - & Género & - & Género & - & Género \\
\hline- & Mujer & - & Mujer & - & Mujer \\
\hline- & Hombre & - & Hombre & - & Hombre \\
\hline- & Sexo & - & Sexo & - & Sexo \\
\hline- & Igualdad & - & Igualdad & - & Igualdad \\
\hline - & Poder & - & Poder & - & Poder \\
\hline - & Cultura & - & Cultura & - & Cultura \\
\hline- & Segregación & - & Segregación & - & Segregación \\
\hline - & Papeles sociales & - & Papeles sociales & - & Papeles sociales \\
\hline- & Equidad & - & Equidad & - & Equidad \\
\hline- & Desigualdad & - & Desigualdad & - & Desigualdad \\
\hline- & Igualdad de género & - & Igualdad de género & - & Igualdad de genero \\
\hline - & Discriminación & - & Discriminación & - & Discriminación \\
\hline - & Femenino & - & Femenino & - & Femenino \\
\hline - & Masculino & - & Masculino & - & Masculino \\
\hline - & Estereotipo & - & Estereotipo & - & Estereotipo \\
\hline - & Diversidad & - & Diversidad & - & Diversidad \\
\hline- & Diferentes & - & Diferentes & - & Diferentes \\
\hline - & Identidad & - & Identidad & - & Identidad \\
\hline - & Tolerancia & - & Tolerancia & - & Tolerancia \\
\hline - & Inclusión & - & Inclusión & - & Inclusión \\
\hline
\end{tabular}

\section{Resultados y discusión}

Para una mejor comprensión de los conceptos, se organizaron los resultados con base en cada una de las competencias de formación en la UCM. En primer lugar se presenta un análisis general de la cantidad de programas que son parte del itinerario formativo de los profesores de educación general básica de esta universidad.

Figura 1. Presentación de porcentajes de programas asociados con cada competencia y su porcentaje de impacto en la formación de profesores de educación general básica con mención.

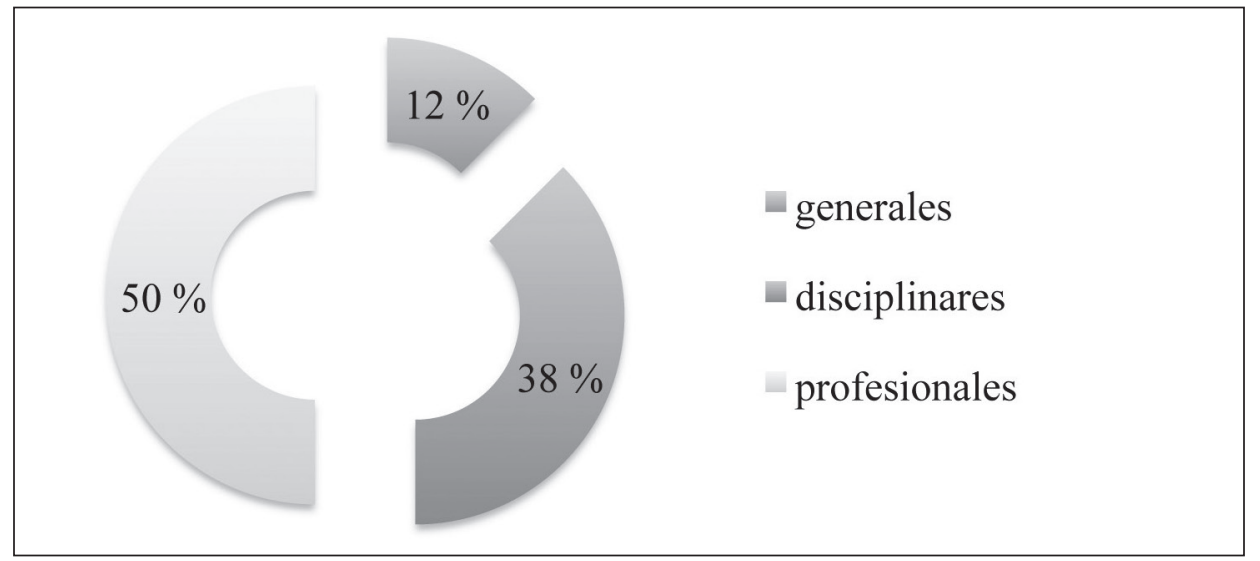


En esta investigación se revisaron 56 programas que son parte del proceso de formación de los profesores de educación básica con mención que han ingresado a estudiar a la UCM a partir del año 2018, ya que las generaciones anteriores fueron formadas bajo otro itinerario. Un $12 \%$ de los programas tributa hacia las competencias generales, destacando que estos programas y módulos son impartidos de manera transversal a todos los profesionales que forma esta universidad; independiente de la Facultad y carrera, para egresar debe haber aprobado los módulos. Un 38 \% de los programas formativos tributan a la competencia disciplinar, encontrándose entre ellos los módulos que forman a los profesores en las especialidades de Lenguaje y Comunicación, Matemática, Ciencias Naturales, Historia, Geografía y Ciencias Sociales. El 50 \% de los programas tributa a la competencia profesional, encontrándose entre ellos los relacionados con las áreas de liderazgo, psicología y evaluación.

Como podemos observar, la formación de profesores que ofrece esta universidad no presenta una uniformidad o equidad en la cantidad de programas asociados con cada una de las competencias. Tobón (2008) destaca la relevancia de la formación bajo este paradigma, planteando que las competencias formalizan los desempeños que se esperan de las personas, debido a que la formación por competencias permite el desarrollo de una educación de calidad.

- La igualdad de género en las competencias generales

En la categoría Competencias generales, dentro del itinerario formativo de PEGB con mención (ver figura 2), se observa que está conformada por los siguientes módulos: Introducción a la Fe y Ética cristiana, Inglés I, Inglés II, Certificación I, Certificación II y Certificación III. De los módulos mencionados anteriormente, solo se pudo analizar los dos primeros, ya que no se tuvo acceso a los otros programas, pero aun así fue posible evidenciar la ausencia de los conceptos relacionados con la igualdad de género, destacando la mayor presencia del concepto de hombre (7) y presentándose el de mujer solo una vez en los dos programas analizados.

Figura 2. Resultado de la presencia de conceptos de igualdad de género en los programas que tributan hacia las competencias generales.

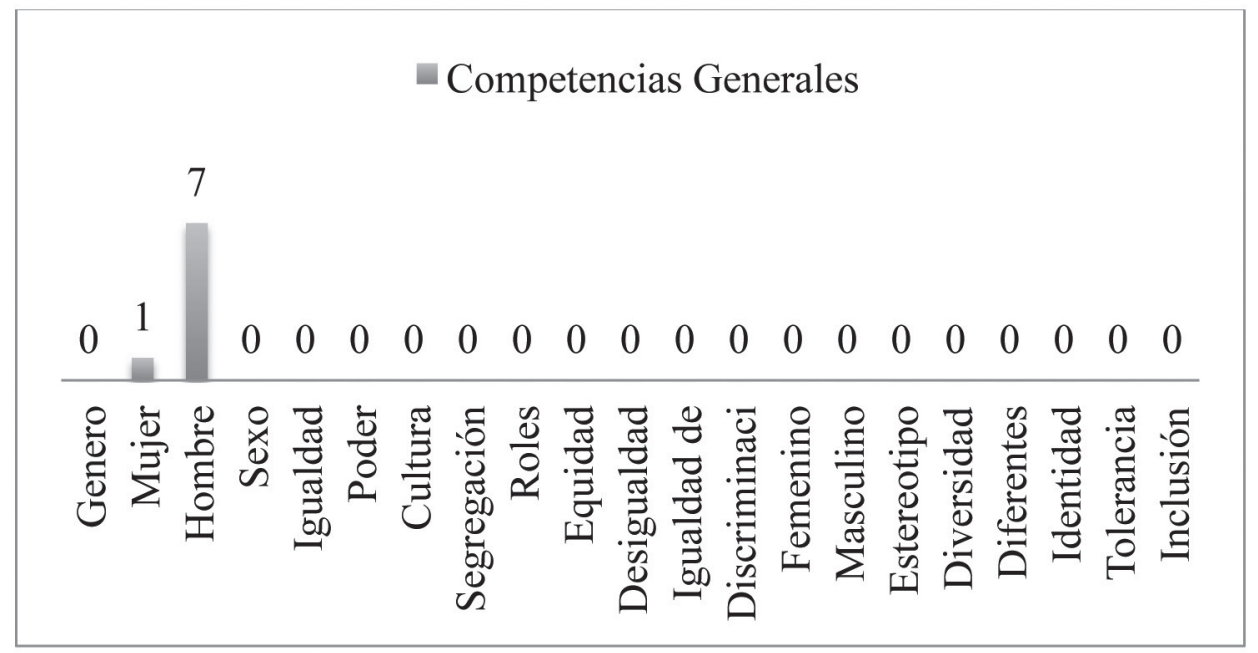

Estos hallazgos demuestran que los esfuerzos que realiza el Ministerio de Educación de Chile (Povedano, Muñiz, Cuesta, \& Musitu, 2015) por difundir el enfoque de género no ha tenido impacto en el itinerario formativo de esta carrera en la UCM. 
- La igualdad de género en las competencias profesionales

La categoría Competencias profesionales presenta 28 programas que tributan al desarrollo de la competencia, donde encontramos programas como Fundamentos Sociológicos de la Educación, Desarrollo Humano e Inclusión Educativa, Orientación Educacional y Vocacional, Gestión Escolar, etc. Como se puede observar en la figura 3 , existe mayor presencia de los conceptos relacionados con la igualdad de género, predominando los conceptos de cultura (9), equidad (8), diversidad (6) y diferentes (6). Si bien se observa un aumento en la frecuencia con que aparecen los conceptos anteriormente mencionados, es necesario recordar que la búsqueda se ha realizado en los 28 programas que tributan a esta competencia, por lo tanto, su frecuencia resulta de poco impacto y no asegura que estos puedan estar presentes en la mayoría de los programas de estudio.

Figura 3. Resultado de la presencia de conceptos de igualdad de género en los programas que tributan hacia las competencias profesionales

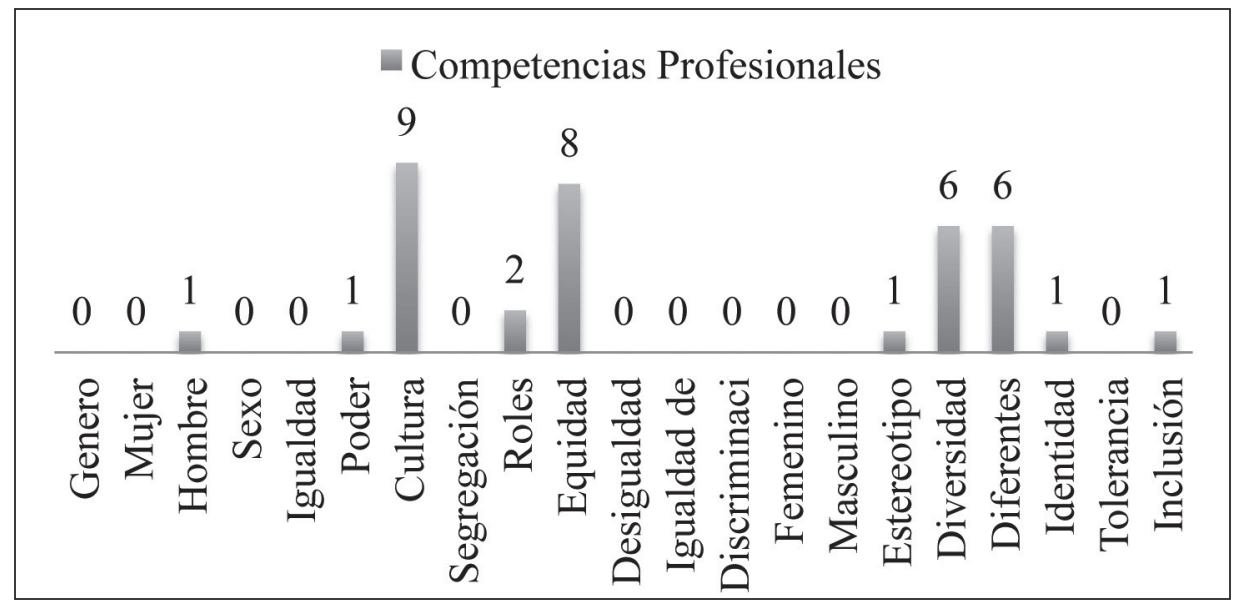

En la actualidad, la equidad y el respeto por la diversidad en educación son temas que se plantean desde el principio de justicia y los derechos humanos (Bartolomé, 2008), es decir, la consideración de estos tópicos en la formación de profesores resulta un tema importante, ya que así ellos identifican el sentido del concepto esto ayudara al desarrollo de prácticas que permiten el logro de una educación acorde a las demandas actuales de igualdad de género.

- La igualdad de género en las competencias disciplinares

La categoría de competencias disciplinares presenta 21 programas que tributan al desarrollo de la competencia, entre ellos están: Didáctica de los números y la Geometría, Lectura y Escritura Inicial, Taller pedagógico, Historia de América y de Chile, etc. Como se puede observar en la figura 4, en estos aumenta la presencia de los conceptos relacionados con la igualdad de género, predominando los conceptos de cultura (13) e igualdad (12), además los conceptos de diversidad, diferentes, tolerancia e inclusión, que se presentan en la misma cantidad (10). Al igual que en las otras dos competencias presentadas anteriormente, el concepto de hombre se presenta con una mayor frecuencia que el de mujer, estableciéndose como un elemento común entre las tres competencias analizadas. 


\section{Figura 4. Resultado de la presencia de conceptos de igualdad de género en los programas que tributan hacia las competencias disciplinares}

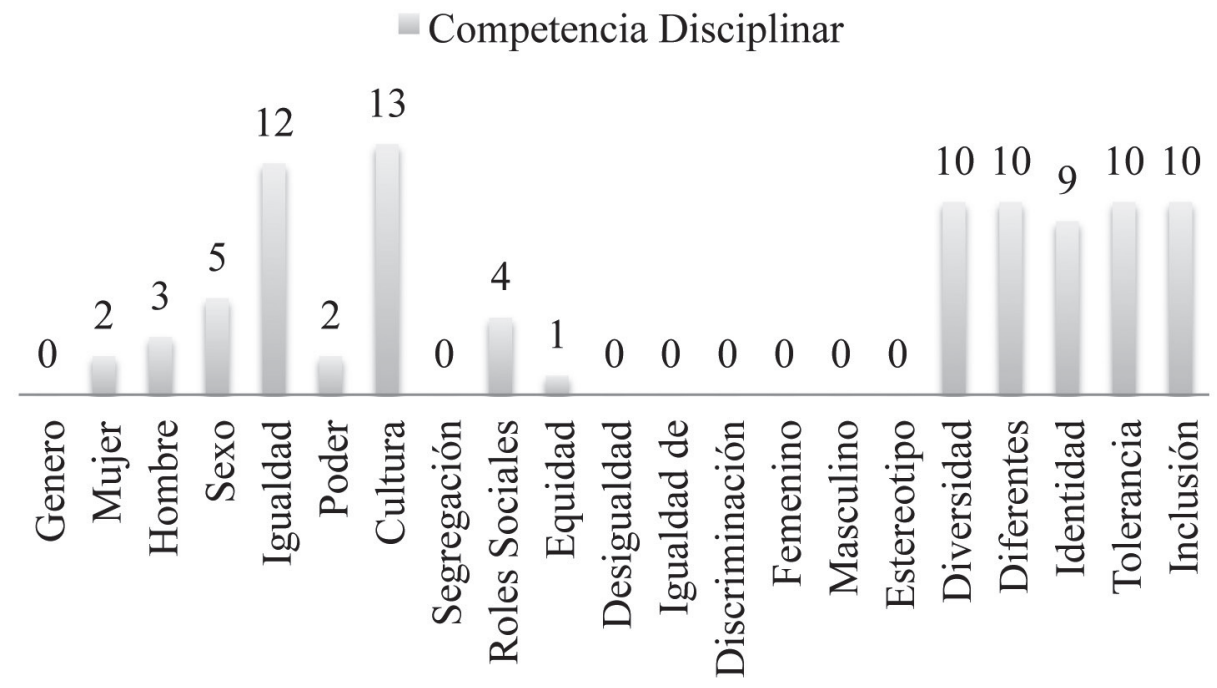

Los contenidos que abordan los programas que tributan a esta competencia, están relacionados con cada de las disciplinas que deberá enseñar el profesor y profesora egresado(a) de esta universidad, por lo tanto, el aumento de la frecuencia de los conceptos relacionados con la igualdad de género resulta importante, ya que, como lo plantean GarcíaPérez, Rebollo, Vega, Barragán-Sánchez, Buzón, \& Piedra, (2011), el profesorado tiene más dificultades para reconocer indicadores relacionados con las políticas de igualdad de género y el lenguaje no sexista, por tanto se hace necesario revertir esto mediante la formación inicial docente.

- La igualdad de género en las competencias generales, disciplinares y profesionales

La figura 5 presenta un resumen de los programas de las tres competencias, predominando el concepto de cultura (21), luego el de igualdad (12), y los de diversidad y diferente se presentan en igual cantidad (16). Como se puede observar en este resumen gráfico, la predominancia del concepto de hombre (14) en relación con el de mujer (2) evidencia que el programa de formación de PEGB en la UCM deja al libre albedrio de los académicos que imparten estos módulos la utilización y profundización de estos conceptos. Esto tiene relación con lo planteado por Coloma (2007), que sostiene que "el lenguaje actúa instrumentalmente, procurando conectar el mundo interno con el externo, a través de la expresión de vivencias personales" (p. 40).

En consecuencia, el dejar en libertad de acción a los académicos para que decidan cómo transmitirán los conceptos y cómo evaluarán las competencias puede producir que estos reproduzcan el tipo de sociedad que ellos consideran válida, limitando a los futuros profesores ya desde su formación. Por lo tanto, los conceptos de igualdad de género no son de difícil verificación en el discurso pedagógico, lo que pone en evidencia que el programa de formación ofrecido por esta universidad perpetúa una educación instrumentalista de la enseñanza, donde el lenguaje (oral o escrito) conforma el medio para transmitir un saber, el cual se encuentra predefinido por la visión y misión de la UCM. 


\section{Figura 5. Resumen de la presencia de conceptos de igualdad de género en los programas que tributan hacia las competencias generales, profesionales y disciplinares}

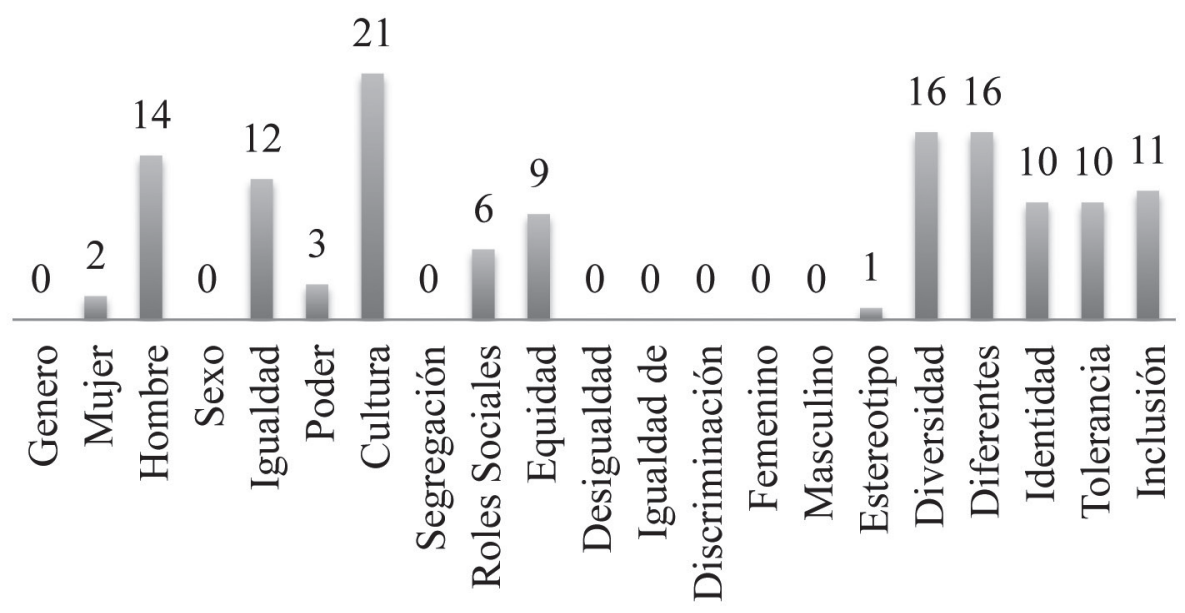

Estos resultados presentan una estrecha relación con otros estudios internacionales relacionados sobre la inclusión de la igualdad de género en los programas de estudio, a su vez estos manifiestan una preocupación sobre todo en el área de educación (Anguita, 2011; Martínez, 2000). Concordando estos con la importancia de visibilizar la igualdad de oportunidades que deben tener los hombres y las mujeres dentro del contexto universitario, asignando al sistema educativo la tarea de corregir o por lo menos desarrollar estrategias para disminuir las desigualdades que existen entre mujeres y hombres en este sentido. Esto hace que sea necesario modificar la dinámica escolar y sus contenidos, para que el sistema educativo deje de ser un reproductor de estereotipos dominantes y de las distintas formas de discriminación vigentes en la sociedad. Este nuevo papel de la escuela resulta de gran importancia, debido a lo planteado por García-Pérez et al (2011), donde expresa que los profesores muestran menos competencias en el reconocimiento de la desigualdad que las profesoras, por lo que esto pudiese ser modificado mediante sistema educativo.

\section{Conclusiones}

Tomando en cuenta que el propósito principal de esta investigación fue indagar sobre la presencia de 21 conceptos relacionados con la igualdad de género, en los programas de formación de la carrera de Pedagogía en Educación General Básica con mención en la Universidad Católica del Maule, se puede concluir, en primer lugar, que los dichos conceptos no se han incorporado de forma significativa en los programas de formación de esta carrera. Si bien existe la presencia de algunos, aún la mayoría de los conceptos no se presentan, mermando las oportunidades que los futuros profesores tendrán de conocer e incorporar en su actuar este tema.

Además, resulta relevante destacar que el itinerario formativo analizado es una nueva propuesta curricular, ofrecida por esta universidad a los estudiantes que ingresan a esta carrera durante el año 2018, la cual presenta una falencia en la igualdad de género, desde las distintas competencias formativas, presentando una desarmonía con las demandas sociales actuales, ya que la perspectiva de género en los últimos años ha cobrado importancia en los ámbitos personal, profesional y, por supuesto, educacional, sobre todo en la formación inicial docente por su papel multiplicador a la hora de su ejercicio docente al interior de las aulas.

Por esto se hace necesario impulsar, dentro de la cultura organizacional de la carrera estudiada, un trabajo colaborativo entre el cuerpo académico para desarrollar un proyecto que responda a las demandas de género desde la mirada de la igualdad entre hombres y mujeres, debido a que es una carrera en que el $84 \%$ de sus estudiantes son mujeres. Por tanto, es una tarea que no puede ser evadida por las autoridades, tanto de la carrera como de la Facultad. Ya que solo así se podrá generar un cambio, en el que los diferentes actores se sientan identificados con el perfil de egreso, donde se plantea que "su ejercicio profesional se orienta por una visión ética e integral del ser humano que contribuya al mejoramiento de la sociedad, a través del 
desarrollo educacional de la región y el país" (Universidad Católica del Maule, 2016).

A su vez, resulta relevante replicar o desarrollar investigaciones que permitan conocer la visión de igualdad de género que se presenta en las otras carreras de la Facultad de Educación tanto de esta casa de estudios como otras que se ubican en la región.

\section{Referencias}

Bartolomé, M. (2008). "Igualdad versus equidad. ¿Enfoques divergentes ante la diversidad educativa?". En F. Etxeberria; I. Sarasola, J.F; Lukas et al. (Coords.), Convivencia, equidad, calidad (155-187). San Sebastián: AIDIPE.

Casal, J., \& Mateu, E. (2003). "Tipos de muestreo". Rev. Epidem. Méd. Prev, 1(1), 3-7.

Coloma, K. (2007). "Racionalidad de la acción que subyace al discurso pedagógico universitario". Revista de Estudios y experiencias en Educación (REXE) 12, 35-51.

García-Pérez, R.; Rebollo, M.Á.; Vega, L.; Barragán-Sánchez, R.; Buzón, O., \& Piedra, J. (2011). "El patriarcado no es transparente: competencias del profesorado para reconocer desigualdad". Cultura y Educación, 23(3), 385-397.

Hernández Sampieri, R.; Fernández Collado, C., \& Baptista Lucio, P. (2014). Metodología de la investigación. 6.a Edición. Editorial McGraw-Hill. México (2014). Hernández, R. Metodología de la Investigación. 6.a Edición, McGraw-Hill, México.

Krippendorff, K. (1990). Metodología del análisis de contenido. Teoría y práctica. Ediciones Paidós. Barcelona.
Martínez, I.Y. (2000). "Sistema sexo/género, identidades y construcción de la subjetividad". Valencia: Universidad de Valencia.

Martínez, R. A. (2011). El reto de la formación del profesorado para la igualdad. Revista electrónica interuniversitaria de formación del profesorado, 14(1), 43-51.

Pinto, M.; Gálvez, C., \& Dijk, T.V. (2018). "Análisis documental de contenido: procesamiento de información".

Povedano, A.; Muñiz, M.; Cuesta, P., \& Musitu, G. (2015). "Educación para la igualdad de género. Un modelo de evaluación". Madrid: FAD.

Rebollar, E. (2013). "El género en los planes de estudio de los grados de educación en las universidades públicas españolas". (Tesis doctoral). Universitat Autónoma de Barcelona, Barcelona, España.

Serrano, G.P. (2016). Diseño de Proyectos Sociales: Aplicaciones prácticas para su planificación, gestión y evaluación (Vol. 12). Narcea Ediciones.

Sotelo, F.S. (2015). El concepto de cultura y los cambios culturales. Sociológica (México), 6(17), 11-25.

Tobón, S. (2008). "La formación basada en competencias en la educación superior: el enfoque complejo". México: Universidad Autónoma de Guadalajara, 5.

Torres, P. (2016). "¿Qué estamos haciendo en Cuba en Evaluación Educativa?". Revista Iberoamericana de Evaluación Educativa, 1(1)

Universidad Católica del Maule (2014). "Proyecto Educativo Institucional". Talca, Chile: [s.n].

Universidad Católica del Maule (2015). "Línea de Fundamentos de la Educación". Talca, Chile: [s.n].

Universidad Católica del Maule (2016). "Proyecto Formativo, Pedagogía en Educación General Básica con Mención". Talca, Chile: [s.n]. 